\title{
Método gráfico para medir la obsolescencia de la literatura de geología: el caso de la Revista Geológica deChile
}

\author{
RubÉN URBIZAGÁSTEGUIALVARADO \\ MARÍA TERESA CORTÉS \\ UniversidaddeCalifornia, \\ Riverside, Riverside,CA 92521, USA \\ ruben@ucrac1.ucr.edu \\ Biblio teca, Servicio Nacio nal deG eo logíayMinería, \\ Av. San taMaría104, San tiago-Chile \\ Bigemi@ reuna.cl
}

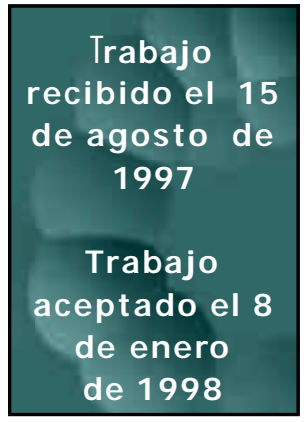

\section{RESUMEN}

Se estudian 1158 citas de documentos publicados en la Revista Gedógica de Chileentre 1995 y 1996 y se aíslan 748 de estas citas como correspondientes a los artículos de revistas. El tamaño de la muestra es de 13\% de todos los artículos publicados en esta revista de 1974 a 1976. De las 194 revistas identificadas, sólo 17 se citan más de diez veces. Para medir la tasa de obsolescencia de esta literatura, se emplea el método gráfico propuesto por Brooks. Se encuentra una alta correlación negativa monotónica de -0.757 entre frecuencia de citación y edad de la literatura citada. También muestra que la $\mathrm{r}^{2}$ es igual a -0.574. El factor anual de envejecimiento es de 0.92 , la vida mediana es de 8.3 años y la vida media de 12 años, respectivamente. Se recomienda quitar de los anaqueles los números envejecidos de esta revista después de 17 años. Tal acción satisfará el 75\% de la demanda posible y deberá satisfacer el restante $25 \%$ a través de la adquisición de fotocopias o recuperando los números viejos del depósito central.

\section{ABST RACT}

Studies 1158 document citations published in the Revista Gedogica deChile between 1995 and 1996 and isolates 748 of these citations as being journal articles. The sample size is $13 \%$ of all articles published in this joumal from 1974 to 1996. Of the 194 individual joumals identified, only seventeen are cited more than ten times. To measure the rate of obsolescence of this literature, the graphic method proposed by Brooks is used. It finds a high monotonic negative correlation of -0.757 between frequency of citation and age of the literature cited. It also shows that the $\mathrm{r}^{2}$ is equal to 0.574 . The annual aging factor is 0.92 , the median life 8.3 
years, and the half-life 12 years respectively. Removal from the shelves the aging issues of this journal after seventeen years is recommended. This action will satisfy 75\% of the possible demand and should satisfy the other $25 \%$ of this demand through the acquisition $t$ of photocopies or by recovering the older issues from a central deposit.

\section{INTRODUCCIÓN}

Todos sabemos que los recursos económicos disponibles en una biblioteca son 1 limitados y por lo tanto no es posible adquirir todas las revistas que uno quisiera poner a disposición de sus usuarios. Ahora bien, se sabe que el uso de los volúmenes retrospectivos de las pocas o muchas revistas adquiridas declinan con el paso del tiempo y la edad. Pero precisamente esos volúmenes envejecidos ocupan espacios en los estantes, y demandan servicios de mantenimiento y reparación que aumentan los costos de administración de la biblioteca. De modo que sería útil poder determinar la tasa de obsolescencia de una revista y usarla en la política de descarte o remoción de la biblioteca. Cuando los usuarios eventualmente solicitasen artículos de esa parte descartada de las revistas, éstas serían recuperadas por medio de la creación y organización de un depósito central, la adquisición (compra) de fotocopias de los artículos solicitados o mediante los préstamos interbibliotecarios preestablecidos o por el sistema de conmutación en la que ésta opera. Por tanto, el problema sería el establecimiento del punto de corte de las revistas destinadas al descarte. Es aquí donde son útiles los estudios de obsolescencia y vida media de la literatura.

La obsolescencia o vida media de la literatura ha sido definida como "el tiempo durante el cual fue publicada la mitad de toda la literatura corrientemente activa" (Burton \& Kebler, 1960). Sin embargo, estrictamente, la obsolescencia se refiere a un fenómeno de descarte y reemplazo. Por ejemplo, hablamos de que alguna cosa es obsoleta cuando ésta ha sido superada y reemplazada por un modelo más eficiente y con mayor capacidad. Pero esto no parece ser cierto para la literatura, pues no sabemos si un ítem de información cualquiera es superado por otro más eficiente. De modo que cuando nos referimos a la obsolescencia de la literatura, en realidad nos estamos refiriendo a una disminución en su frecuencia de uso o citación, pero no a su descarte definitivo. Es por eso que Diodato (1994) se refiere a la obsolescencia como "el decrecimiento en el uso de un documento o grupo de documentos conforme el documento envejece." Ciertamente, la literatura del pasado se revisa y actualiza, los hechos ya conocidos sobre un fenómeno son incorporados y fusionados con los nuevos conocimientos sobre ese mismo fenómeno, por lo tanto, son escritos nuevamente y reinterpretados en términos de nuevas teorías, como correcciones y refinamientos de los artículos pu- 
blicados y puestos en circulación por las revistas científicas, pero no sabemos si definitivamente esta literatura es "obsoleta."

En una carta dirigida al editor de la revista Jaumal of Doumentation, Brookes (1975) señalaba que a pesar de que el principal objetivo de los estudios de obsolescencia era optimar la selección y descarte de los documentos que ocupan espacios medibles en centímetros en los estantes de una biblioteca, no había sido posible desarrollar una técnica estandarizada de trabajo. Sin embargo, ya por esa época, Brookes había desarrollado esa técnica y desde 1970 venía publicando una serie de trabajos dirigidos a proporcionar a los bibliotecarios un modelo muy sencillo para medir la obsolescencia de la literatura y así facilitar el descarte o remoción (Brookes, 1970a; Brookes, 1970b; Brookes, 1972; Brookes, 1973). En estos trabajos insistía en las siguientes condiciones para medir la tasa de obsolescencia de una literatura:

a) D efinirbibliográficamenteel cam po dees tu dio, el asun to, los usuariosy losintereses de los usuarios donde se aplicaran las medidas. Enfatizaba el hecho de que "[...]cualquier bibliotecario preocupado con la tasa de obsolescencia de cual quieradelasrevistascien tíficas existentes en susestan tes, no puedeconfiar enlasmedidasderivadasdeotrasbiblio tecasaun quelasco rrija. Elbiblio tecario debe medir directamente el uso [hecho] en su propia biblioteca" (Brookes, 1970b).

b) D efinir el tamaño de la muestra de la cual serán derivadas las medidas. En este punto hay contradicciones en sus recomendaciones. En su trabajo inicial recomienda una muestra de 580 ítems y afirmaba que este tamaño garantizaría un 95\% de resultados confiables, i.e un margen de error del 5\% (Brookes, 1970a). Y aen un artículo posteriorllamabalaaten ción parala"necesidad deestablecerel tamaño delamues trausadademodo quelasignifican ciadelasdiferen cias pueda ser probada estadísticamente" y recomendaba“[...]una muestra de al menos400 referencias para producir estimados de la vida media que puedan ser confiables so brelabasedel 10\% desuvalorreal" (Broo kes, 1970b). Posteriormen teafirma ba que "la técnica más directaparamedirlaob so lescen cianecesi tadeunamues tradealmenos2000 citasdelaliteraturadelasun to publicado en un determinado año" (Brookes, 1973).

c) Usar también el concepto de utilidad de la literatura. Recuerden que el envejecimiento de los volúmenes de una revistaA quepro du ce 200 artículosporaño en un determinado cam po, siem preserádosvecesmásútil quelarevistaB, queproduce 100 artículosporaño (entérminosdepro babilidades)ysupo nien do quelas dosrevistassean usadas en el mismo con tex to. Lautilidad deunarevistaes definida como el número de referencias que se es pe ra que sele ha gan y ten gan en el contex to deunabiblio tecaparticularduran teel tiem po desu existen cia. Engene ral, sesupo nequelautili dad decadafas cícu lo decaeráen lamismatasa o pro porción, en ton ces, lamejorpo líticaserádescartarigualestasasdeutilidad. Tam bién 
llamaba la atención para "la necesidad de estudios empíricos cuidadosamente controlados para probar las suposicionesso bre las que se basan los análisis y resultados teóricos de los estudios de obsolescencia" (Brookes, 1970b).

A pesar de que existen muchos estudios de citación, en general, éstos han estado enfocados a la identificación de revistas individuales más frecuentemente citadas, pero pocos estudios han sido conducidos en relación con el descarte o remoción a depósitos locales o regionales vía la identificación de la tasa de obsolescencia de la literatura. Estos estudios han estado confinados al campo de la genética (Stinson \& Lancaster, 1987); y del arroz (Zhang, 1992) y ninguno ha estudiado específicamente el campo de la geología. Esta carencia y las posibilidades que ofrece el método gráfico propuesto por Brookes nos impulsó a experimentarlo en el campo de la geología chilena.

\section{MATERIAL Y MÉTODO}

Como unidades de análisis fueron seleccionadas las citas bibliográficas existentes en los artículos publicados por la Revista Gedógca deChile Esta revista es editada por el Servicio Nacional de Geología y Minería con la colaboración de la Sociedad Geológica de Chile y comenzó a ser publicada en 1974. En estos 22 años de actividad ininterrumpida, esta revista ha publicado un total de 23 volúmenes con 45 fascículos, debido a que algunos fueron publicados como número doble, que conjuntamente produjeron 307 artículos, que citaron un total de 9.058 documentos. Un resumen detallado de esta producción puede ser observado en la Tabla 1. A partir de 1988 la Revista Gelógica deChilecambió su numeración simple correlativa, que llegó hasta el número 31, por una numeración compuesta iniciada en el volumen 15. Como sólo estamos interesados en medir la obsolescencia sincrónica, de este total de 23 volúmenes, fueron seleccionados para análisis los volúmenes 22 y 23 correspondientes a los años 1995 y 1996. En este periodo se publicaron 32 artículos que citaron 1158 fuentes que representan una muestra del 13\% del total de citas. También se observó que en este periodo hubo una media de 7 artículos por fascículo, con una media de 30 citas por artículo. Esto indica un alto consumo aparente de literatura como refuerzo a la publicación de nuevos artículos. O bsérvese también que el volumen de las citas aumentó en las épocas más recientes y hubo una concentración del 53\% del total de las citas en los últimos 9 años (de 1988 a 1996).

Para medir la tasa de obsolescencia de la Revista Gedóġa deChilese usó el método gráfico propuesto por Brookes(1970a, 1970b), pero se utilizaron sólo las 748 citas encontradas en artículos de revistas, por tanto se descartaron las citas encontradas en anales de congresos, libros, tesis, mapas y publicaciones en prensa. Estas 748 citas representan una muestra de 8.3\% en relación con el total de 
las citas encontradas en los 22 años de existencia de la revista y de 65\% en relación con el total de citas verificadas en los volúmenes 22 y 23 aquí estudiadas.

Tabla 1

\begin{tabular}{|c|c|c|c|c|}
\hline \multicolumn{5}{|c|}{$\begin{array}{l}\text { Producción de artículos de la ReistaGelóǵca deChile, } \\
\qquad 1974-1996\end{array}$} \\
\hline Años & Vols. & Números & No. de Artículos & No. de Citas \\
\hline 1974 & & 1 & 9 & 250 \\
\hline 1975 & & 2 & 6 & 88 \\
\hline 1976 & & 3 & 3 & 92 \\
\hline 1977 & & 4 & 4 & 98 \\
\hline 1978 & & $5-6$ & 10 & 139 \\
\hline 1979 & & $7-8$ & 9 & 282 \\
\hline 1980 & & $9-11$ & 17 & 493 \\
\hline 1981 & & $12-14$ & 15 & 444 \\
\hline 1982 & & $15-17$ & 14 & 422 \\
\hline 1983 & & $18-20$ & 14 & 316 \\
\hline 1984 & & $21-23$ & 19 & 541 \\
\hline 1985 & & $25-26$ & 19 & 475 \\
\hline 1986 & & $27-29$ & 15 & 332 \\
\hline 1987 & & $30-31$ & 15 & 274 \\
\hline 1988 & 15 & $1-2$ & 15 & 497 \\
\hline 1989 & 16 & $1-2$ & 14 & 534 \\
\hline 1990 & 17 & $1-2$ & 12 & 571 \\
\hline 1991 & 18 & $1-2$ & 16 & 419 \\
\hline 1992 & 19 & $1-2$ & 17 & 577 \\
\hline 1993 & 20 & $1-2$ & 13 & 392 \\
\hline 1994 & 21 & $1-2$ & 19 & 665 \\
\hline 1995 & 22 & $1-2$ & 18 & 689 \\
\hline 1996 & 23 & $1-2$ & 14 & 468 \\
\hline TOTAL & 23 & 49 & 307 & 9.058 \\
\hline
\end{tabular}




\section{RESULTADOS}

La clasificación por tipos de fuentes de las 1158 citas estudiadas se muestra en la Tabla 2. Como se puede observar, los autores que publicaron sus artículos en la Revista Gedógica deChileen el periodo de 1995-1996 basaron sus trabajos en artículos de revistas y alcanzaron una relación de 4:1 para las citas de anales de congresos; 5:1 para las citas de libros; y 15:1 para las citas de tesis. Las citas a mapas y documentos en proceso de publicación fueron de poca recurrencia.

Tabla 2

\begin{tabular}{|c|c|c|}
\hline \multicolumn{3}{|c|}{ CIT ACIONES CLASIFICADAS SEGÚN TIPO DE MATERIAL } \\
\hline Tipo de fuente & No. de citaciones & Porcentaje \\
\hline Revistas & 748 & 64.6 \\
\hline Congresos & 171 & 14.8 \\
\hline Libros & 165 & 14.2 \\
\hline Tesis & 51 & 4.4 \\
\hline Mapas & 7 & 0.6 \\
\hline En prensa & 16 & 1.4 \\
\hline TOTAL & 1158 & 100.0 \\
\hline
\end{tabular}

Las 748 citas de artículos de revistas nos permitieron identificar un total de 194 revistas, que ordenadas en forma descendente de acuerdo con su frecuencia de citación están listadas en el Anexo, pero sólo aquellas revistas que fueron citadas 5 o más veces en el periodo estudiado. Una evaluación más detallada de estas revistas está en proceso y servirá de base para otro trabajo. Sin embargo, es fácil notar que existe un grupo de 17 revistas de alta citación. Esto indica que estas 17 revistas son las que en el periodo estudiado atrajeron mayor atención de los investigadores de geología en Chile.

Un análisis del grupo tipificado como Congresos, que incluye las actas de congresos, simposios, mesas redondas, reuniones (nacionales e internacionales), mostró que de las 175 citaciones encontradas en este tipo de material, 45\% era de las actas del Congreso Geológico Chileno, y 19\% de las actas del Congreso Geológico Argentino; el resto se dispersaba en una serie de congresos con poco impacto: Congreso Latinoamericano de Paleontología, Congreso Latinoamericano de G eología y la Reunión Argentina de Sedimentología. Aquí hay que resaltar que las fronteras más largas que tiene Chile son con Argentina y posiblemente este hecho hace que compartan estas preocupaciones geológicas con los congresos habidos en ese país fronterizo, y claro, sin perder de vista lo que sucede en el área en Latinoamérica pero con muy poca incidencia. 
La distribución de la frecuencia de citas a artículos de revistas aparecidas en la Rexista Gelóġa deChile 1995-1996, según la edad de la literatura citada se muestra en la Tabla 3. La agrupación por quinquenios permite observar que conforme crece la edad de la literatura disminuye el volumen de citación. En los tres primeros quinquenios se realizan aproximadamente $70 \%$ de las citas, i.e70\% de la literatura citada tiene 15 años de edad o menos y 80\% de esta literatura tiene menos de 20 años. Sin embargo, se debe notar que $54 \%$ de la literatura citada tiene menos de 10 años de edad y que más o menos $25 \%$ de esta literatura tiene menos de 5 años.

Tabla3

\begin{tabular}{|c|c|c|c|}
\hline \multicolumn{4}{|c|}{ DISTRIBUCIÓN DE LA FRE CUENCIA DE CITASSE GÚN } \\
LAEDAD DE LA LITE RATU RACITADA \\
\hline Edad & Frecuencia de citas & \% & $\sum$ \% \\
\hline $0-4$ & 177 & 23.7 & 23.7 \\
\hline $5-9$ & 225 & 30.0 & 53.7 \\
\hline $10-14$ & 114 & 15.3 & 69.0 \\
\hline $15-19$ & 82 & 10.9 & 79.9 \\
\hline $20-24$ & 50 & 6.7 & 86.6 \\
\hline $25-29$ & 40 & 5.4 & 92.0 \\
\hline $30-34$ & 13 & 1.7 & 93.7 \\
\hline $35-39$ & 15 & 2.0 & 95.7 \\
\hline $40+$ & 32 & 4.3 & 100.0 \\
\hline TOTAL & 748 & 100.0 & \\
\hline
\end{tabular}

La Tabla 4 muestra los datos de la distribución de la edad de la literatura versus la frecuencia de citación, usada para calcular el factor de envejecimiento de la Reista Gelógica deChile Se encontró que el rango de la edad corría retrospectivamente desde 1996 hasta 1896 y el rango de la frecuencia de citación iba de 1 cita en el nivel más bajo hasta 70 citaciones en el más alto. Se optó por cortar y acumular los datos referentes a la edad de la literatura y su frecuencia de citas cuando éstos alcanzaron la edad de 56 años, es decir, en 1940.

La Figra 1 muestra el gráfico de barras de las citas a revistas encontradas en el periodo estudiado. La Figura 2 muestra el histograma de frecuencias de las mismas. Al observar tanto el gráfico como el histograma se puede afirmar que ocurre una acumulación inicial en los primeros años de edad y que crece paulatinamente en los primeros cuatro años (más rápido en los primeros dos años y más lento en los dos posteriores) hasta alcanzar su máxima expresión en el quinto año. Esto indica que llevaría aproximadamente casi 5 años para que los 
88 Innestigadón Biblideedógica v. 12 No. 24 enero/ junio de1998

nuevos artículos publicados en la Reista Gęóġa deChilesean difundidos, conocidos, incorporados en nuevos artículos y reflejados como citaciones en los nuevos artículos publicados en sus páginas, por los geólogos chilenos. También se observa que ocurren algunas fluctuaciones de la disminución uniforme esperada. Si estas fluctuaciones son aleatorias o si representan verdaderas características del envejecimiento de la revista, sólo se podrá determinar tomando más muestras de citaciones y observando si se repiten de la misma manera 0 cambian de características.

Tabla 4

\begin{tabular}{|c|c|c|c|}
\hline \multicolumn{4}{|c|}{$\begin{array}{c}\text { Distribución de la fre cuen ciadecitasapareci dasen la } \\
\text { Reista Gelógica deChile, 1995-1996 }\end{array}$} \\
\hline Año & Edad (t) & Frecuenciadecitas & Citas en la cola $T(t)$ \\
\hline 1996 & 0 & 2 & 748 \\
\hline 1995 & 1 & 24 & 746 \\
\hline 1994 & 2 & 45 & 722 \\
\hline 1993 & 3 & 51 & 677 \\
\hline 1992 & 4 & 55 & 626 \\
\hline 1991 & 5 & 70 & 571 \\
\hline 1990 & 6 & 39 & 501 \\
\hline 1989 & 7 & 29 & 462 \\
\hline 1988 & 8 & 47 & 433 \\
\hline 1987 & 9 & 40 & 386 \\
\hline 1986 & 10 & 21 & 346 \\
\hline 1985 & 11 & 26 & 325 \\
\hline 1984 & 12 & 28 & 299 \\
\hline 1983 & 13 & 14 & 271 \\
\hline 1982 & 14 & 25 & 257 \\
\hline 1981 & 15 & 19 & 232 \\
\hline 1980 & 16 & 25 & 213 \\
\hline 1979 & 17 & 13 & 188 \\
\hline 1978 & 18 & 12 & 175 \\
\hline 1977 & 19 & 13 & 163 \\
\hline 1976 & 20 & 9 & 150 \\
\hline 1975 & 21 & 10 & 141 \\
\hline 1974 & 22 & 9 & 131 \\
\hline 1973 & 23 & 13 & 122 \\
\hline 1972 & 24 & 9 & 109 \\
\hline 1971 & 25 & 10 & 100 \\
\hline
\end{tabular}


Tabla 4

(continuacoón)

\begin{tabular}{|c|c|c|c|}
\hline \multicolumn{4}{|c|}{$\begin{array}{l}\text { Dis tri bu ción dela fre cuen ciadeci tas apa re ci das en la } \\
\text { ReistaGelóǵca deChile, 1995-1996 }\end{array}$} \\
\hline Año & Edad (t) & Frecuencia de citas & Citas en la cola $T(t)$ \\
\hline 1970 & 26 & 8 & 90 \\
\hline 1969 & 27 & 8 & 82 \\
\hline 1968 & 28 & 9 & 74 \\
\hline 1967 & 29 & 5 & 65 \\
\hline 1966 & 30 & 4 & 60 \\
\hline 1965 & 31 & 2 & 56 \\
\hline 1964 & 32 & 1 & 54 \\
\hline 1963 & 33 & 3 & 53 \\
\hline 1962 & 34 & 3 & 50 \\
\hline 1961 & 35 & 3 & 47 \\
\hline 1960 & 36 & 3 & 44 \\
\hline 1959 & 37 & 2 & 41 \\
\hline 1958 & 38 & 5 & 39 \\
\hline 1957 & 39 & 2 & 34 \\
\hline 1956 & 40 & 2 & 32 \\
\hline $1955+$ & $41+$ & 3 & 30 \\
\hline 1954 & 42 & 2 & 27 \\
\hline 1953 & 43 & 1 & 25 \\
\hline 1952 & 44 & 1 & 24 \\
\hline 1951 & 45 & 1 & 23 \\
\hline 1950 & 46 & 0 & 22 \\
\hline 1949 & 47 & 2 & 22 \\
\hline 1948 & 48 & 1 & 20 \\
\hline 1947 & 49 & 0 & 19 \\
\hline 1946 & 50 & 1 & 19 \\
\hline 1945 & 51 & 0 & 18 \\
\hline 1944 & 52 & 1 & 18 \\
\hline 1943 & 53 & 1 & 17 \\
\hline 1942 & 54 & 1 & 16 \\
\hline 1941 & 55 & 1 & 15 \\
\hline 1940 & 56 & $14+$ & 14 \\
\hline Total & & 748 & \\
\hline
\end{tabular}


Figura1

Gráfico de ba ras de lafre cuen cia de ci tas versus la edad de la li te ra tu ra

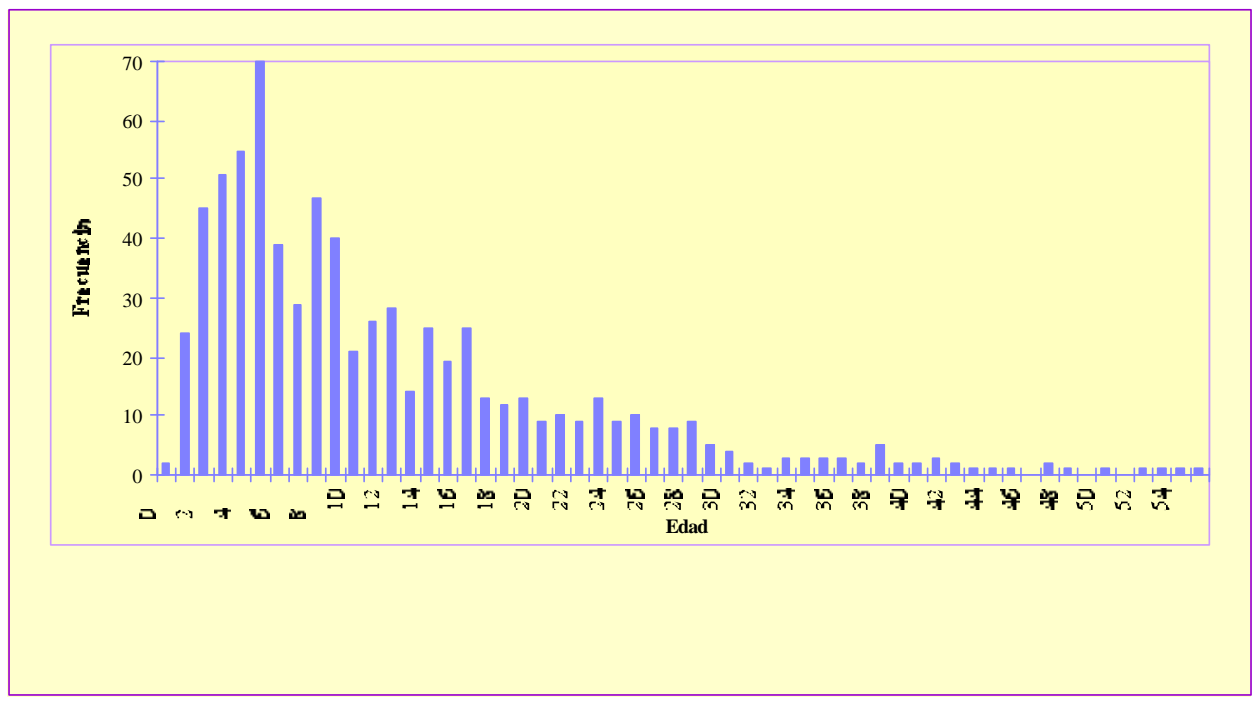

Figura2

His to gra ma de la fre cuen cia de ci tas versus la edad

delaliteraturacitada

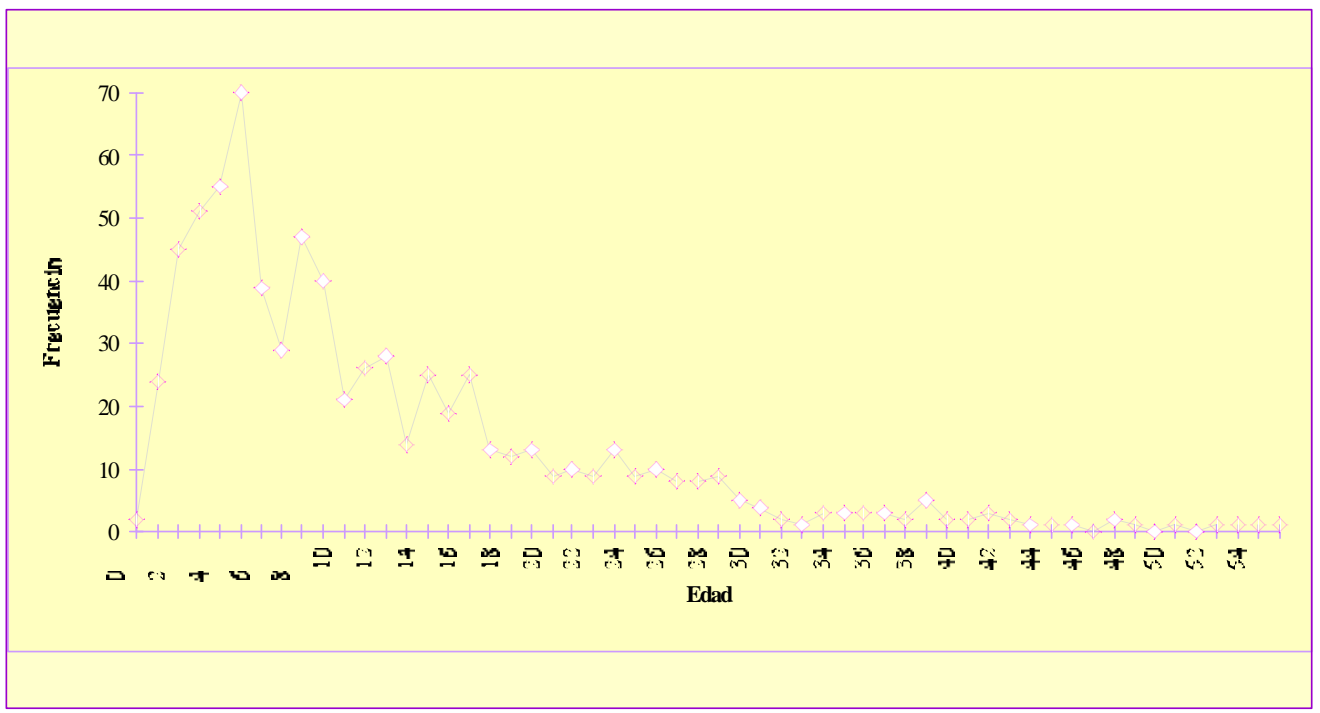


La Figura 3 muestra el diagrama de dispersión de la frecuencia acumulada versus la edad de las citas en un gráfico linear. Puede observarse que existe una caída aproximadamente exponencial conforme la literatura citada envejece y es evidente una tendencia en la que los altos valores de la frecuencia de citación están asociados con la edad de la literatura más reciente, y los valores más bajos de la frecuencia de citación están asociados con la edad de la literatura más reciente, y los valores más bajos de la frecuencia de citación están asociados con las edades más antiguas de la literatura citada. Esta figura, pues, nos proporciona una indicación visual de que la frecuencia de citación y la edad de la literatura citada covarían negativamente. También se encontró que entre la edad de la literatura y la frecuencia de citación existe una alta correlación monotónica negativa de -0.757 y que la constante a es igual a 33.9 y la caída de la línea recta de regresión b es igual a -0.742. Este resultado produce una ecuación matemática de la forma:

$$
\mathrm{y}=33.9-0.742 \mathrm{x}
$$

donde yes la frecuencia de citas a los artículos de revistas en la Reista Gedóġa deChiley x es la edad de los artículos de las revistas citadas. Esta ecuación reafirma que existe una relación negativa entre ambas variables.

Fig. 3

Diagramadedispersión delafrecuen ciaacumula da ver sus la edad de las ci tas

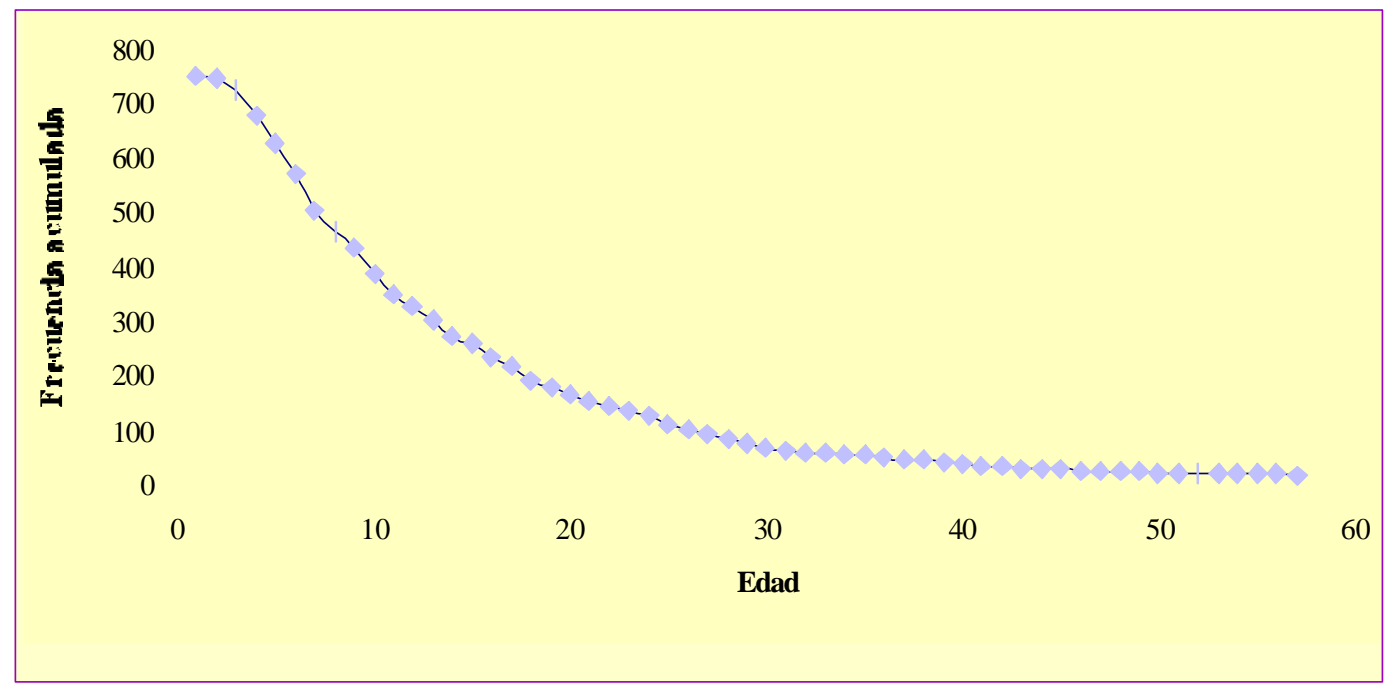

El coeficiente de determinación $r^{2}$ encontrado fue de - 0.574 con un error estándar de 10.74 , lo que indica que $57.4 \%$ de las variaciones en la frecuencia de citación depende de las variaciones encontradas en la edad de la literatura. Indica 
además que entre ambas variables existe una fuerte asociación linear negativa. El test estadístico $\mathrm{F}$ encontrado fue de 73.65 que indica que entre ambas variables hay una co-asociación que es explicada en $74 \%$ de sus variaciones. También el test t encontrado fue de 12.07 al nivel de 4.3 de significación. Como vemos, ambos tests indican un buen ajuste del modelo asociativo.

La Figra 4 muestra la recta de regresión en una escala semi-logarítmica. Cuando se calculó la correlación entre el logaritmo de los valores de citación y la edad de la literatura se encontró que también existe una alta correlación monotónica negativa de -0.075. También se encontró que la constante a fue igual a 6.566 y la caída de la recta de regresión b fue igual a -0.075. Este resultado produce una ecuación matemática de la forma:

$$
1 \text { ny }=6.566-0.075 \mathrm{x}
$$

donde yes el valor del logaritmo de base 10 de los valores de la frecuencia de citación y x es la edad de la literatura citada. El r² encontrado fue de 0.9890 e indicó que 98\% de las variaciones en la frecuencia de citación depende de las variaciones encontradas en la edad de la literatura. Entre estas variables existe, pues, una alta asociación linear.

Siguiendo las propuestas de Brookes (1973) para calcular el factor de envejecimiento anual de la Revista Gedógica deChile, los valores acumulados de la columna 4 de la Tabla 4 fueron trazados sobre un papel semi-logarítmico de 3 ciclos. Este trazado puede ser observado en la Figura 5, donde la recta es aproximadamente linear de 1996 a 1971, acumulándose en este periodo

Fig 4

Rectaderegresión delascitasacumuladas (escalalogańtmica)versus la edad (escalalinear)

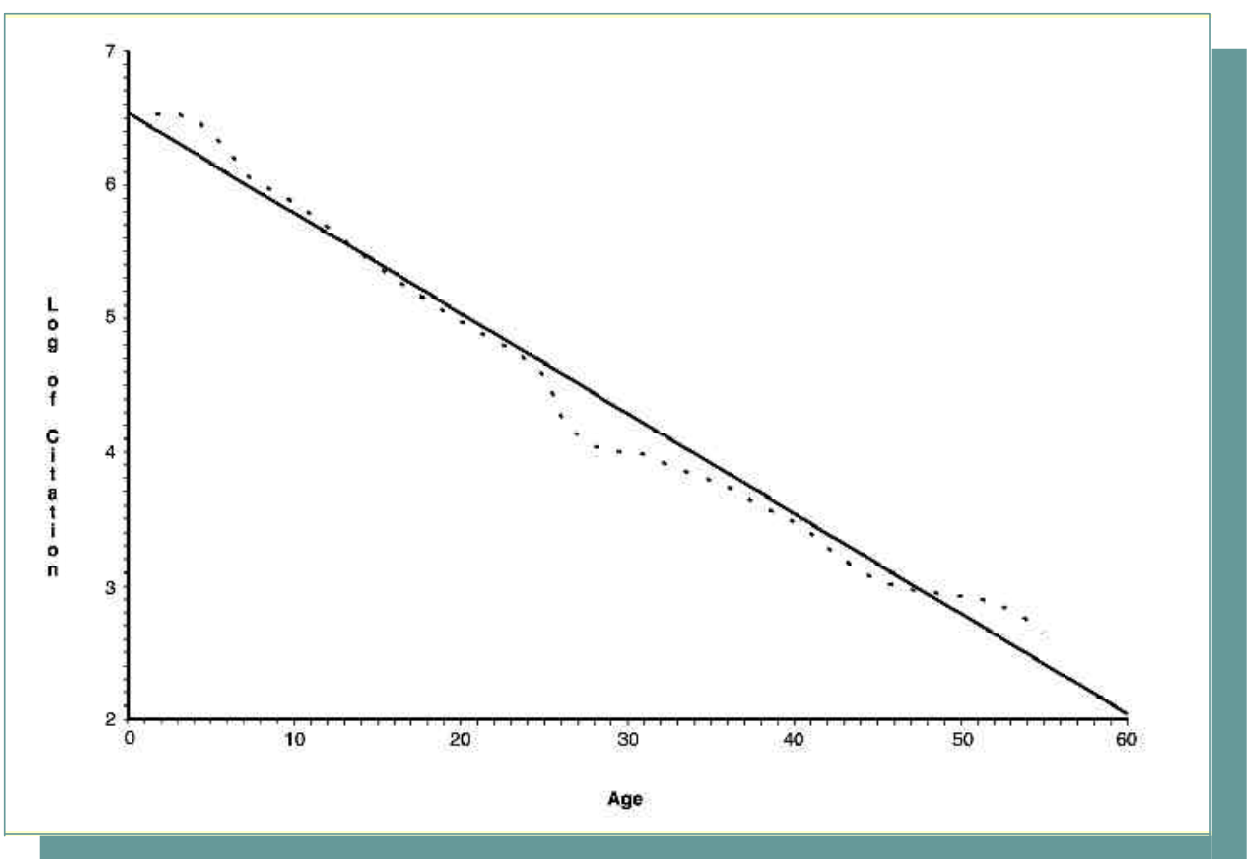


Método gráfico para medir la obsolescencia de la literatura de geología... 93

Fig 5

Gráfico semi-logańtmico de los datosdela frecuencia

acumulada(escalalogańtmica) versus la edad de las ci tas (esca la li near)

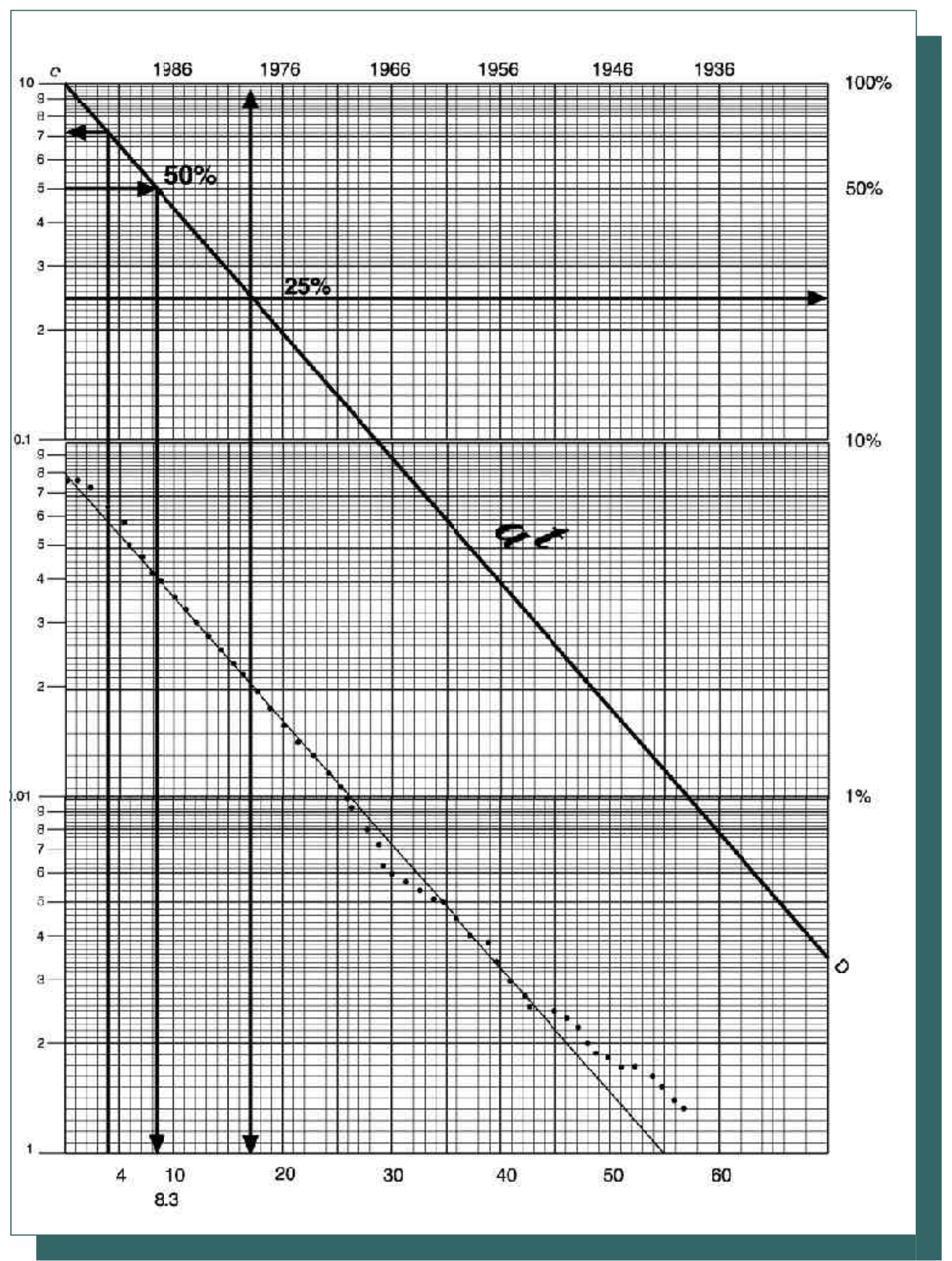


aproximadamente $88 \%$ de las citas. Paralela a esta recta, se trazó otra recta CD, desde el tope del lado izquierdo del papel semi-logarítmico. Si se usa esta recta $C D$, se puede leer que $a^{4}$ es más o menos igual a 0.72 . Por tanto, tenemos que:

$$
\begin{aligned}
& a^{4}=0.72 \\
& a^{2}=0.848 \\
& a=0.92
\end{aligned}
$$

Puede observarse de igual manera que la semi-vida hes igual a más o menos 8.3 años. Este valor indica que el uso de la literatura geológica para 1988 había caído hasta el 50\% de su total y puede ser leído directamente de la línea paralela CD. Un mejor cálculo puede obtenerse observando la edad en que las citas descienden hasta el $25 \%$ de su total, un periodo que corresponderá a 2 semi-vidas. En este caso CD cruza el nivel del 25\% a los 17 años, lo que confirma el cálculo anterior. Se observa también que $72 \%$ de las citas tiene menos de 15 años de edad. Las medidas ay hestán relacionadas y deben satisfacer la ecuación:

$$
\mathrm{a}^{\mathrm{h}}=\frac{1}{2}
$$

de modo que:

$$
\begin{gathered}
\mathrm{a}^{\mathrm{h}}=0.5 \\
\mathrm{~h} \log \mathrm{a}=0.5 \\
\mathrm{~h}=\frac{\log 0.5}{\log 0.92} \\
\mathrm{~h}=\frac{-0.30}{-0.04} \\
\mathrm{~h}=8.313 \text { años. }
\end{gathered}
$$

Otro método para calcular el factor de envejecimiento anual sugerido por Brookes (1973) es dividir la muestra en dos grupos. De modo que el primer grupo estuvo compuesto por las citas con hasta 8 años de edad con un total de $\mathrm{m}=362$. El segundo grupo estuvo compuesto por aquellas citas con 9 y más años de edad con un total de $n=386$. Para calcular el factor de envejecimiento a se usó la siguiente fórmula:

$$
\mathrm{a}^{8}=\frac{\mathrm{n}}{\mathrm{m}+\mathrm{n}}
$$

de modo que 


$$
\begin{gathered}
a^{8}=\frac{386}{362+386} \\
a^{8}=\frac{386}{748} \\
a^{8}=0.52 \\
a^{4}=0.72 \\
a^{2}=0.85
\end{gathered}
$$

$\mathrm{a}=0.92$. Éste es un valor constante para todos los valores de la edad, i.e. cuando las citaciones alcanzan una edad de 1, 2, 3, 4, 5, ... taños de edad.

Para calcular la semi-vida se usó la fórmula:

$$
\mathrm{a}^{\mathrm{h}}=\frac{1}{2}
$$

de modo que:

$$
\begin{aligned}
& (0.92)^{\mathrm{h}}=0.5 \\
& \mathrm{~h} \log 0.92=\log 0.5 \\
& \mathrm{~h}=\frac{\log 0.5}{\log 0.92} \\
& \mathrm{~h}=\frac{-0.30}{-0.04} \\
& \mathrm{~h}=8.313 \text { años }
\end{aligned}
$$

Para calcular la vida media se usó la siguiente fórmula:

$$
\begin{aligned}
& 1 / \alpha=\log (1 / a) \\
& 1 / \alpha=\log (1 / 0.92) \\
& 1 / \alpha=\log (1.087) \\
& 1 / \alpha=0.083 \\
& \alpha=1 / 0.083 \\
& \alpha=11.993 \text { años, es decir } \approx 12 \text { años. }
\end{aligned}
$$

Como se ve, ambos métodos producen los mismos resultados. Para calcular el factor de utilidad de la revista se usó la fórmula: 


$$
\begin{aligned}
& \mathrm{U}=\frac{1}{(1-\mathrm{a})} \\
& \mathrm{U}=\frac{1}{(1-0.92)} \\
& \mathrm{U}=\frac{1}{0.08} \\
& \mathrm{U}=12.5
\end{aligned}
$$

Este valor indica apenas que la Reista Geológica deChileenvejece lentamente, casi a una tasa de 10\% al año y es casi similar a la diferencia de 1-0.92 en relación con el factor de envejecimiento, esto significa que, como esta revista envejece lentamente, tendrá la posibilidad de atraer más citas por parte de sus usuarios.

La suma total de citas que la Revista Geeớgica deChileatraerá en toda su vida activa de 22 años, fue calculada usando la fórmula:

$$
\begin{gathered}
\mathrm{U}=\frac{\text { Tdal deditas }}{1-\mathrm{a}} \\
\mathrm{U}=\frac{748}{1-0.92} \\
\mathrm{U}=\frac{748}{0.08} \\
\mathrm{U}=9350
\end{gathered}
$$

Esta cifra indica que se espera que la revista, en sus 45 fascículos publicados en estos 22 años de actividad continua, haya tenido un total de hasta 9350 citas. Si comparamos esta cantidad total esperada con la cantidad total observada que es igual a 9.058 citas, se nota que existe una coincidencia bastante aproximada, pues hay solamente una diferencia del 3\%.

\section{CONCLUSIÓN}

En esta investigación fueron estudiadas las fechas de citación de 1158 referencias bibliográficas y de 748 citas hechas a títulos de revistas. El tamaño de la muestra de 13\% fue adecuado al estudio, y el espacio de tiempo de 22 años fue lo suficientemente largo como para determinar una tasa de obsolescencia de la literatura de geología, pues esta literatura como cualquier otra, también envejece. Como era de esperarse para esta clase de distribución, se pudieron identificar tres medidas diferentes: el factor de envejecimiento anual, que fue de 0.92; la semi-vida 0 mediana, de 8.3 años y la vida media, de 12 años. Esta vida media es muy 
semejante a los 11.8 años encontrados por Burton \& Kebler (1960) para la literatura de geología americana. De modo que, para ganar espacio en los estantes de la biblioteca, sin pérdida significativa de capacidad de atención, sería posible remover de los estantes aquellos fascículos poco usados de la Reista Gedóǵca de Chile con más de 12 años de edad. Sin embargo, adoptando una actitud más conservadora y prudente, se podría ampliar este punto de corte hasta los 17 años; con este punto de corte, la revista aún estaría en condiciones de atender $75 \%$ de la demanda y sólo $25 \%$ de esta demanda sería recuperada eventualmente del lugar donde fueron depositados los fascículos obsolescentes. Se observó también que los artículos publicados en esta revista llevan más o menos entre 3 y 5 años para ser difundidos, incorporados como conocimiento aprovechable y ser citados en nuevos artículos publicados en la misma revista.

El método gráfico propuesto por Brookes y probado en este trabajo parece producir buenos resultados. Sin embargo, sería interesante examinar este método también en otras áreas para observar si producen los mismos resultados. También fue posible identificar un grupo de 17 revistas de frecuente consulta por parte de los geólogos chilenos, pero esto es tema de otro estudio.

\section{BiBLIOGRAFÍA}

Burton, R. E. \& Kebler, R. W. "The "half-life" of some cientific and technical lite ratures." AmeicanDoamettanion, 11:18-22, 1960.

BRO o KES, B. C. "Obsolescence: letter to the editor." Jamal of Doumentation, 34(1): 36-47, 1975.

- -. "O bsolescence of special library periodicals: sampling errors and utility contours." Jaumal of theAmeican Sociey for Infomation Saiene 21: 320-329, Sept. 1970.

- -. "The growth, utility and obsolescence of scientific periodical literature." Jaumal of Doumentation, 26(4): 283-294, D ec. 1970.

- - . "The aging of scientific literature." Prodens of InfomationSaience colletion of papes/ edited by A. I. Cherny. Moscow: International Federation of Documentation, Study Committee "Research on Theoretical Basis of Information," 1972. pp. 6690.

- -. "Numerical methods of bibliographical analysis." Library trends 18-43, July 1973.

Dio D A T, V. "D ictionary of bibliometrics." New York: The Haworth Press, 1994.

Stinson, E. R. \& F. W. Lancaster. "Synchronous versus diachronous methos in the measurement of obsolescence by citation studies." Jaumal of Information Saence, 13(2): 65-74, 1987.

ZHANG, Q. "The literature of rice: a bibliometrics survey." IAALD Quartety, 37(3): 151-158, 1992. 
98 Investigadón Biblideedógica V. 12 No. 24 enero/ junio de1998

ANEXO

\section{REVISTASCITADAS ORDENADAS \\ EN FORMADESCEN DENTE}

\begin{tabular}{|c|c|c|}
\hline Orden & Título & $\begin{array}{c}\text { Frecuen } \\
\text { cia de Ci- } \\
\text { tación }\end{array}$ \\
\hline 1 & Reista Gedógica deChile & 59 \\
\hline 2 & Gedory & 39 \\
\hline 3 & Jaumal of Geephysical Research & 38 \\
\hline 4 & Jamal of SathAmeicanEarthSaienes & 35 \\
\hline 5 & Bulletin(Gedogical Soidy of Ameria) & 27 \\
\hline 6 & Contributions toMinealogyan Petrogy & 22 \\
\hline 7 & EconamicGelogy & 21 \\
\hline 8 & EarthandPlandary SaieneLdtes & 20 \\
\hline 9 & Carta Gelógica deChile(SevidoNac DeGedoǵa) & 20 \\
\hline 10 & Tetongphysics & 17 \\
\hline 11 & Ameghiama & 16 \\
\hline 12 & ReistaAsociaión Gelógica Argentima & 16 \\
\hline 13 & Special Paper (Geelogical Sociey of Amrica) & 13 \\
\hline 14 & Jaumal ar theGedogical SoidyofLandon & 12 \\
\hline 15 & Bdefín(InstitutodeInvesticaciones Gelógicas) & 12 \\
\hline 16 & Nature & 11 \\
\hline 17 & Tetorics & 11 \\
\hline 18 & Ada Geodimica et Cosmohimica & 9 \\
\hline 19 & Bulletin (Amrican Assciation of PerdermGedogists) & 8 \\
\hline 20 & Comnicaiones (Univ. deChileDeta deGedoǵa) & 8 \\
\hline 21 & GedojsheRundshau & 8 \\
\hline 22 & Jaumal of Seelimentary Pexdogy & 8 \\
\hline 23 & Jamal of Petrdogy & 7 \\
\hline 24 & Reista Chilena deHistoria Natural & 7 \\
\hline 25 & Ameican Minealogist & 6 \\
\hline 26 & Ameican Jaumal of Saience & 6 \\
\hline 27 & EarthSaieneReiens & 6 \\
\hline 28 & Chemical Gedogy & 5 \\
\hline 29 & Jamml of V dcandogy and Gethemml Resentch & 5 \\
\hline 30 & Bdéín(MuseoNacional deHistoria Natural) & 5 \\
\hline 31 & Sedimetdogy & 5 \\
\hline 32 & Saiere & 5 \\
\hline
\end{tabular}

